\title{
Microarthridion corbisierae sp. nov. (Harpacticoida, Tachidiidae), um novo copépode da meiofauna do litoral norte do estado de São Paulo, Brasil
}

\author{
Terue C. Kihara ${ }^{1,2} \&$ Carlos E. F. Rocha ${ }^{1}$ \\ ${ }^{1}$ Departamento de Zoologia, Instituto de Biociências, Universidade de São Paulo. Rua do Matão, Travessa 14, 321, \\ 05508-900 São Paulo, Brasil. E-mail: tkihara@ib.usp.br \\ ${ }^{2}$ Centro Universitário Adventista de São Paulo. Estrada de Itapecerica 5859, 05858-001 São Paulo, Brasil.
}

\begin{abstract}
Microarthridion corbisierae sp. nov. (Harpacticoida, Tachidiidae), a new meiofaunal copepod from the Northern coast of São Paulo State, Brazil. During studies on the diversity of meiobenthic marine harpacticoid copepods, a new representative of the family Tachidiidae was found on the inner continental shelf of São Paulo State, between São Sebastião Channel and Ubatumirim Bay, Ubatuba $\left(23^{\circ} 24^{\prime} S, 44^{\circ} 57,6^{\prime} \mathrm{W}\right)$. Complementary material was collected near Enseada Beach, Ubatuba $\left(23^{\circ} 30^{\prime} \mathrm{S}, 45^{\circ} \mathrm{O} 5^{\prime} \mathrm{W}\right)$. Although the new species shares with M. laurenticum (Nicholls, 1940) the reduced antennules, it can be easily distinguished from its congeners by leg 4 endopod with only two segments and the reduction of the number of inner setae of legs 1-4 endopod-3 and leg 3 exopod-3. Tachidiids, typically inhabiting fine sediments of brackish and marine shallow waters in the Northern Hemisphere, are good indicators of heavy organic pollution. This is the first record of the family Tachidiidae in the southern hemisphere.
\end{abstract}

KEY WORDS. Continental shelf; Copepoda; meiobenthos; taxonomy.

RESUMO. Durante estudos sobre a diversidade de copépodes harpacticóides meiobentônicos marinhos, um novo representante da família Tachidiidae foi encontrado na plataforma continental interna do estado de São Paulo, entre o canal de São Sebastião e a área costeira em frente á praia de Ubatumirim, Ubatuba ( $\left.23^{\circ} 24^{\prime} \mathrm{S}, 44^{\circ} 57,6^{\prime} \mathrm{W}\right)$. Material complementar foi coletado próximo à praia da Enseada, Ubatuba $\left(23^{\circ} 30^{\prime} \mathrm{S}, 45^{\circ} \mathrm{O} 5^{\prime} \mathrm{W}\right)$. Embora esta nova espécie compartilhe com M. laurenticum (Nicholls, 1940) a redução das antênulas, ela pode ser facilmente distinguida de seus congêneres pela presença de apenas 2 artículos no endópodo da perna 4 e pela redução no número de cerdas internas do endópodo-3 das pernas 1-4 e do exópodo-3 da perna 3. Os Tachidiidae, que tipicamente habitam sedimentos finos de águas rasas salobras e marinhas do Hemisfério Norte, são considerados bioindicadores de ambientes ricos em material orgânico. Este é o primeiro relato da família no hemisfério sul.

PALAVRAS-CHAVE. Copepoda; meiobentos; plataforma continental; taxonomia.

A família Tachidiidae, cujos representantes estão entre os copépodes mais comuns em águas costeiras européias, possui 14 espécies distribuídas por seis gêneros, segundo Huys et al. (2005). SEIFried (2003) propôs a sinonímia de Euterpinidae com Tachidiidae por ambos os grupos compartilharem as mesmas sinapomorfias. A composição de Tachidiidae, então, seria de sete gêneros e 15 espécies. G. Boxshall não aceita tal proposição (Boxshall \& Halsey 2004), o que é seguido neste artigo.

Os gêneros Geeopsis Huys, 1996 e Cithadius Bowman, 1972 são representados por uma espécie cada: Geeopsis incisipes (Klie, 1913) e Cithadius cyathurae Bowman, 1972.

Huys et al. (2005) estudaram mais detalhadamente o gênero Tachidius Lilljeborg, 1853. A espécie Tachidius (Tachidius) vicinospinalis Shen \& Tai, 1964 foi revisada e passou a ser a espécie-tipo do gênero Sinotachidius Huys, Ohtsuka, ConroyDalton \& Kikuchi, 2005. Já Tachidius discipes Giesbrecht, 1881 sensu Song \& Chang 1995, foi considerada species inquirendae de Sinotachidius, pela falta de informações sobre os machos. Neotachidius, originalmente proposto como um subgênero por SHEN \& TAI (1963), foi elevado à categoria de gênero para incluir T. (N.) triangularis Shen \& Tai, 1963, como espécie-tipo, e mais duas novas espécies, $N$. coreanus e $N$. parvus.

Microarthridion Lang, 1944, o gênero mais diversificado, possui sete espécies (Boxshall \& Halsey 2004), não levando em conta a sinonímia de M. perkinsi Bodin, 1970 com M. reductum (Monard, 1935), como sugerido por Huys et al. (1996). Microarthridion possui representantes na costa leste da América do Norte (Nicholls 1940), ao longo de praticamente toda a costa européia (Veldre \& Maemets 1956, Dussart 1967, Lorenzen 1969, Bodin 1970, Huys et al. 1996) e em águas costeiras da China e Coréia (Shen \& TAI 1973).

Os Tachidiidae tipicamente habitam sedimentos finos de

Revista Brasileira de Zoologia 24 (4): 907-918, dezembro 2007 
águas rasas salobras e marinhas do hemisfério norte, sendo considerados bioindicadores de ambientes ricos em material orgânico (Huys et al. 1996). Este é o primeiro relato da família no hemisfério sul.

\section{MATERIAL E MÉTODOS}

O material foi coletado pelo Departamento de Oceanografia Biológica, Instituto Oceanográfico, Universidade de São Paulo (DOB-IOUSP), durante a realização do projeto multidisciplinar "Utilização Racional do Ecossistema Costeiro da Região Tropical Brasileira: Estado de São Paulo", que teve por objetivo investigar a plataforma continental interna do litoral norte do estado de São Paulo, entre a ilha de São Sebastião, ao sul, e a enseada de Ubatumirim, Ubatuba, ao norte (CorbISIER 1993).

As 12 estações de coleta encontram-se entre as isóbatas de 15 e $50 \mathrm{~m}$. A principal característica oceanográfica que estrutura o ecossistema ao largo de Ubatuba é a sazonalidade da penetração de águas frias da Água Central do Atlântico Sul (ACAS) (Pires-Vanin \& Matsuura 1993). A área investigada possui considerável variedade de tipos sedimentológicos, mas há o domínio de areias finas e muito finas (Furtado \& Mahiques 1990). Detalhes da metodologia de coleta e de análise dos parâmetros ambientais são dados por CORBISIER (1993).

Os Tachidiidae aqui estudados foram encontrados a 19 $\mathrm{m}$ de profundidade. $\mathrm{O}$ sedimento era composto por areia muito fina e moderadamente selecionada. A temperatura de fundo era de $15,2^{\circ} \mathrm{C}$ e a salinidade, $35,5 \mathrm{ppm}$. Os teores de carbono orgânico e carbonatos biodetríticos foram de 0,6\% e 10,0\%, respectivamente. Material complementar foi obtido na viagem de coleta da disciplina "Ecologia de micro e meiobentos marinhos", do curso de pós-graduação do Departamento de Oceanografia Biológica do IOUSP, em 1999, em estação próxima à praia da Enseada, Ubatuba, entre o costão e a ponta da Espia $\left(23^{\circ} 30^{\prime} \mathrm{S}, 45^{\circ} 05^{\prime} \mathrm{W}\right)$. O material foi coletado em areia fina, a uma profundidade de $7 \mathrm{~m}$.

Espécimes inteiros foram examinados em preparações temporárias. O material foi montado em ácido lático a $80 \%$ e fragmentos de lamínulas serviram como suporte para a lamínula de cobertura da preparação. Após o exame, os espécimes foram acondicionados em álcool (etanol) a 70\%. Nas montagens semipermanentes, animais inteiros ou dissecados foram montados em glicerina entre lâmina e lamínula; essas preparações foram seladas com Glyceel ${ }^{\circledR}$.

Para a análise do material e preparação das ilustrações, utilizou-se microscópio óptico Zeiss Axioskop 2 Plus com contraste de interferência diferencial, dispositivo fotográfico digital Nikon Coolpix $995^{\circledR}$ e câmara clara acoplados.

Lotes de espécimes foram depositados no Museu de Zoologia (MZUSP) e no Departamento de Zoologia do Instituto de Biociências da Universidade de São Paulo (DZ-IBUSP).

Para facilitar a compreensão do texto descritivo apresentamos as seguintes definições: a) janelas tegumentares - áreas bem definidas, em que a cutícula é menos espessa; b) antênula quirócera - condição da antênula do macho em que a geniculação ocorre entre um artículo bastante alargado e esclerotizado e o artículo terminal (Fig. 24).

\section{TAXONOMIA}

\section{Microarthridion corbisierae sp. nov.}

\section{Figs $1-28$}

Localidade tipo. Plataforma continental interna do lito-

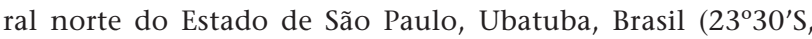
$\left.45^{\circ} 05^{\prime} \mathrm{W}\right)$.

Material examinado. Brasil, São Paulo: Ubatuba (enseada de Ubatumirim, $23^{\circ} 24^{\prime}$ S, $44^{\circ} 57,6^{\prime} \mathrm{W}$ ), 1 fêmea, III.1989, equipe do DOB-IOUSP leg.; 4 fêmeas e 4 machos adultos (praia da Enseada, $23^{\circ} 30^{\prime} \mathrm{S}, 45^{\circ} 05^{\prime} \mathrm{W}$ ), 5.X.1999, turma da disciplina "Ecologia de micro e meiobentos marinhos" leg., DOB-IOUSP. Holótipo (MZUSP 16465) e 2 parátipos (MZUSP 16466), todos inteiros, depositados no Museu de Zoologia. Material montado em lâminas, na coleção do DZ-IBUSP.

Descrição. Fêmea (Figs 1-21). Comprimento total do corpo, medido da região anterior do rostro até a margem posterior do ramo caudal, 270-320 $\mu \mathrm{m}(\mathrm{n}=5$, média = 284). Prossomo: urossomo 1,3-1,5:1 ( $\mathrm{n}=5$, média $=1,4: 1)$. Corpo de forma ciclopóide: prossomo e urossomo claramente distintos (Figs 1 e 2). Cefalotórax com janela tegumentar dorsal; pares de janelas tegumentares laterais presentes no cefalotórax e nos segmentos portadores das pernas 2-4. (Figs 1 e 2). Segmentos do corpo com fileiras transversais de espínulos; franjas hialinas ao longo dos bordos posteriores dos segmentos corporais pouco desenvolvidas e levemente espinulosas (Figs 1 e 2).

Segmento genital duplo (Figs 1-4) com articulação original entre o segmento genital propriamente dito e o primeiro segmento abdominal visível dorsalmente (Fig. 3) e marcada por espessamentos quitinosos internos laterais e ventrolaterais; fileiras de espínulos nas superfícies dorsal, ventrolateral e ventral, exceto na área genital. Área genital (Figs 4 e 21) com gonóporos abrindo-se em fenda transversal sem ornamentos; poro copulador situado logo no início da metade posterior do segmento. Segmento anal (Figs 5 e 6) curto, com opérculo espinuloso.

Ramo caudal (Figs 1 e 4-6) curto, 1,2 vezes mais largo que longo, com fileira diagonal de espínulos no lado interno e fileira de espínulos ao longo da margem distal do ramo; extensão ventral do bordo posterior recobrindo as bases das cerdas IV, V e VI (Fig. 4). Cerda I reduzida e posicionada ventro-lateralmente na metade do ramo, aproximadamente; cerda II lisa e implantada dorso-lateralmente próximo à cerda VII; cerda III unipinada no lado externo; cerdas IV unipinada e V bipinada, separadas entre si e com planos de fratura pré-estabelecidos; cerda VI bipinada; cerda VII lisa e triarticulada na base.

Rostro (Fig. 7) curto, cônico e sem definição na base; pares de sensilas dorsais e apicais; poro dorsal mediano.

Antênula (Figs 7 e 8) curta, com cinco artículos; artículo1 com fileiras transversais de espínulos na superfície anterior. Fórmula de cerdas e estetos: 1, 7, 8 + esteto, 1, 13 + esteto. 


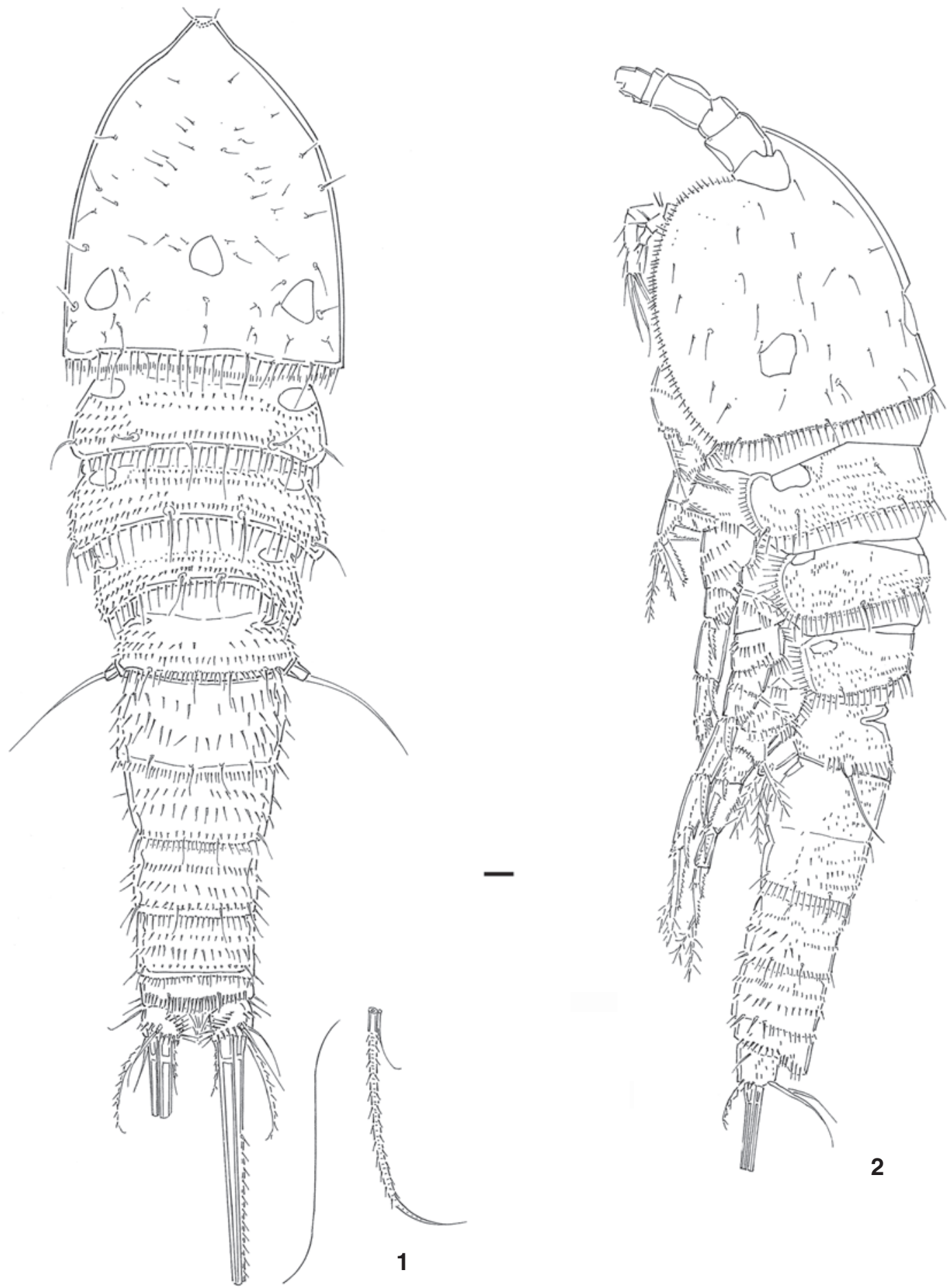

Figuras 1-2. Microarthridion corbisierae sp. nov., fêmea: (1) hábito, dorsal; (2) hábito, lateral. Escala = $10 \mu \mathrm{m}$.

Antena (Fig. 9). Base com fileiras de espínulos nos lados externo e interno. Exópodo com dois artículos; exópodo-1 com duas cerdas bipinadas; exópodo-2 com três cerdas bipinadas, uma lateral interna e duas apicais. Endópodo com dois artículos; endópodo-1 com fileira de espínulos longos e delgados; endópodo-2 com fileiras de espínulos; região média interna com 

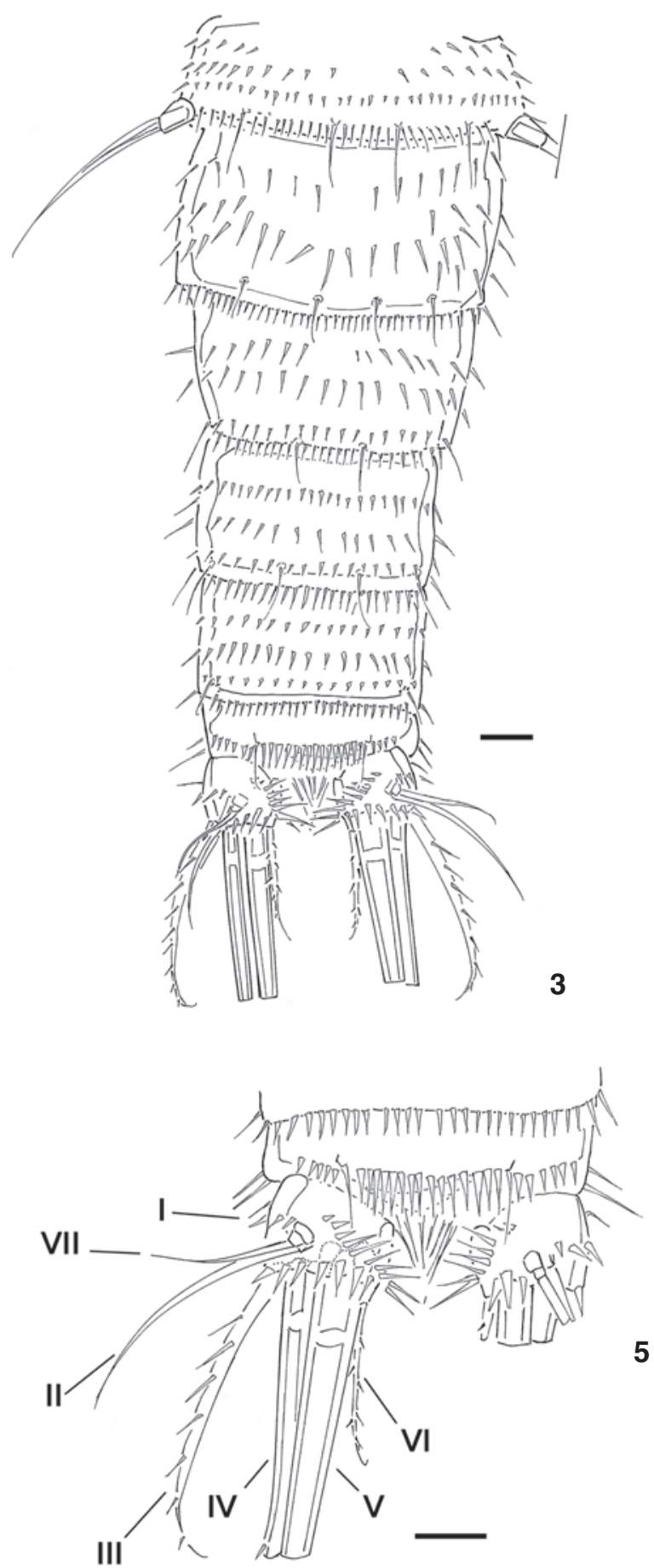

3

5
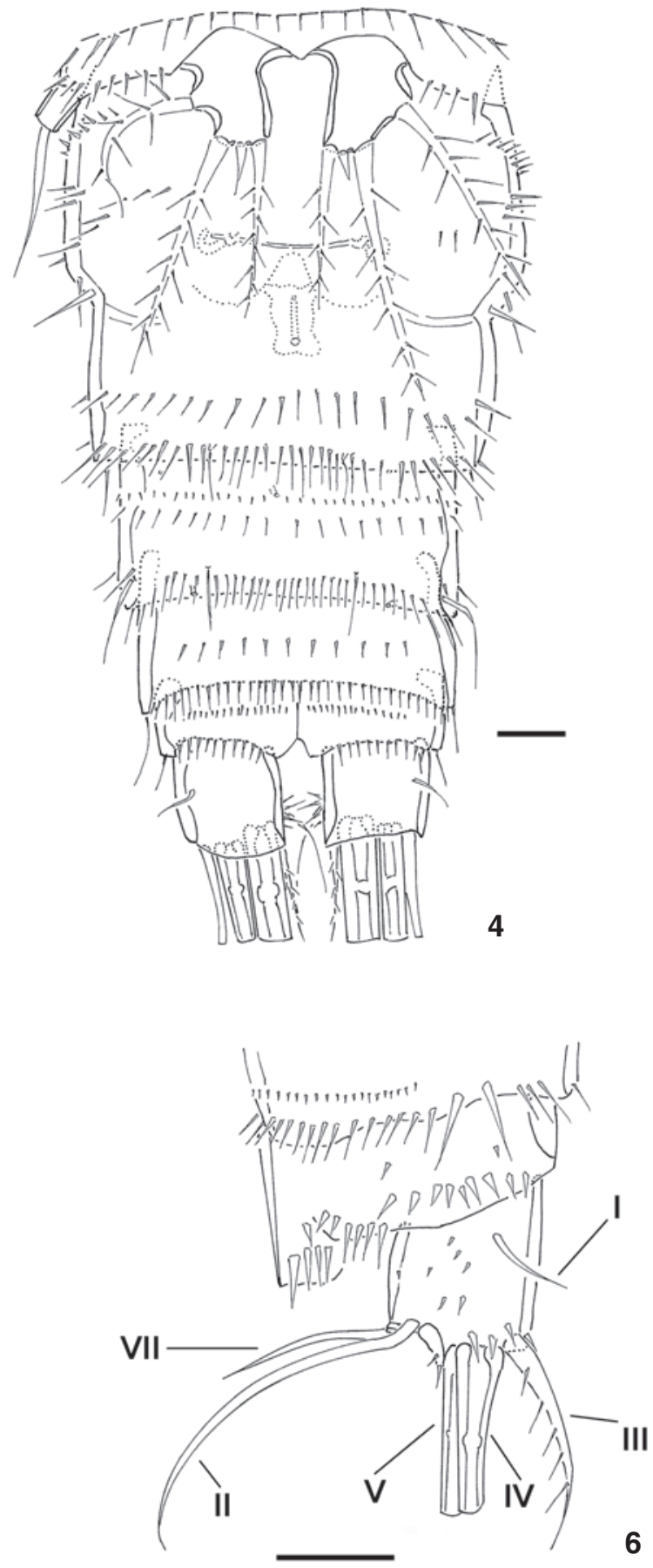

Figuras 3-6. Microarthridion corbisierae sp. nov., fêmea: (3) urossomo, dorsal; (4) urossomo, ventral; (5) segmento anal e ramos caudais com as cerdas I - VII, dorsal; (6) segmento anal e ramo caudal (cerda VI não representada), lateral. Escala = $10 \mu \mathrm{m}$.

três cerdas pinadas; ápice com cinco cerdas pinadas (duas simples e três geniculadas) e uma lisa.

Labro (Fig. 10) bem desenvolvido, triangular; região distal com par de protuberâncias, poro mediano e margens serrilhadas. Mandíbula (Fig. 11). Coxa robusta; gnatobase com projeções dentiformes desiguais e cerda bipinada. Base com cerda 


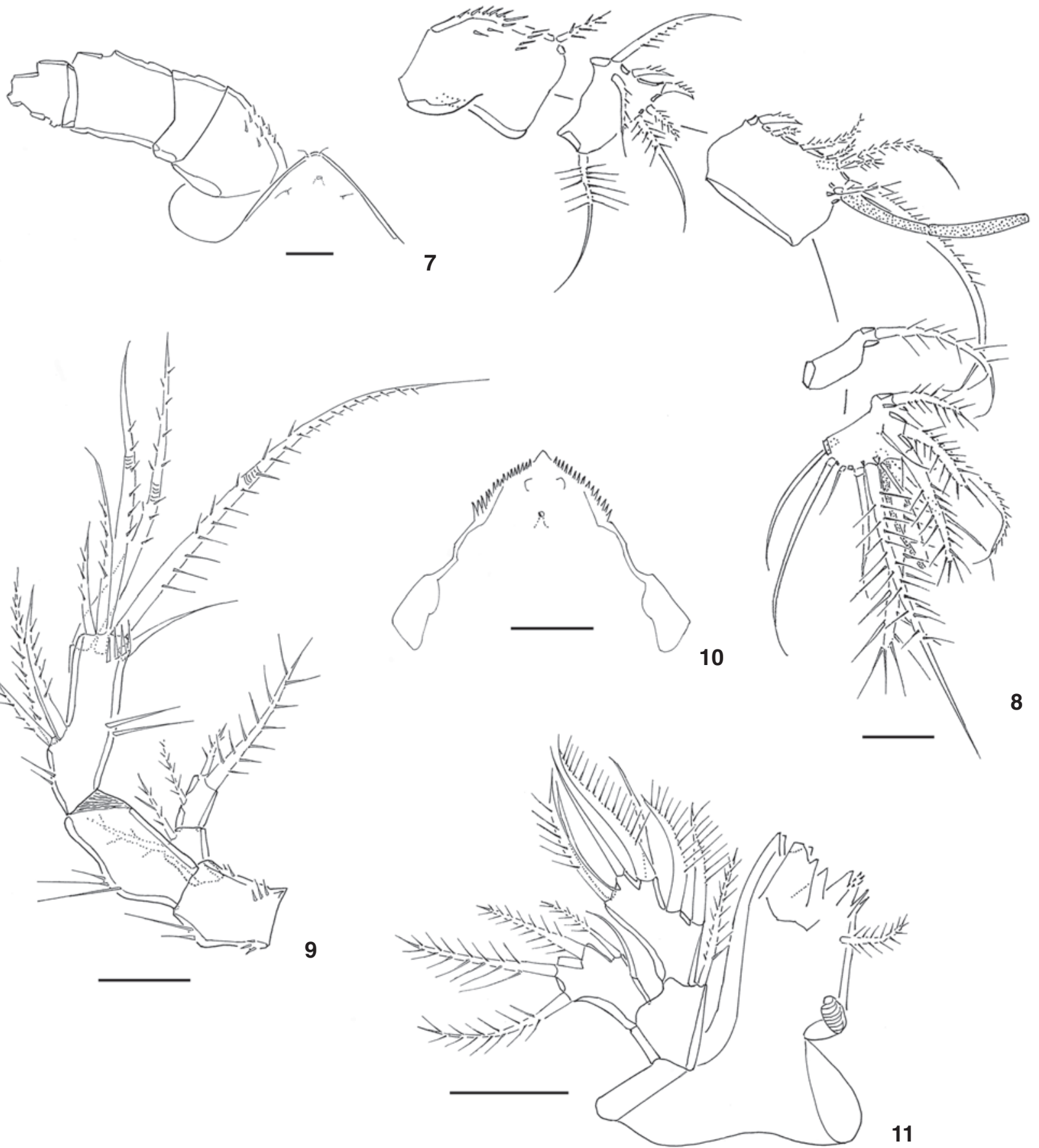

Figuras 7-11. Microarthridion corbisierae sp. nov., fêmea: (7) rostro e antênula esquerda (cerdas e outros elementos da ornamentação da antênula foram omitidos), dorsal; (8) antênula, dorsal (os artículos estão ilustrados separadamente); (9) antena; (10) labro; (11) mandíbula. Escala $=10 \mu \mathrm{m}$.

bipinada. Exópodo menor que endópodo, com uma cerda lisa e quatro cerdas bipinadas. Endópodo com duas cerdas unipinadas laterais; cinco cerdas (três lisas e duas unipinadas) na porção apical.

Maxílula (Figs 12 e 13). Artrito da pré-coxa com 10 espinhos curvos e pontiagudos, uma cerda unipinada e duas cerdas 


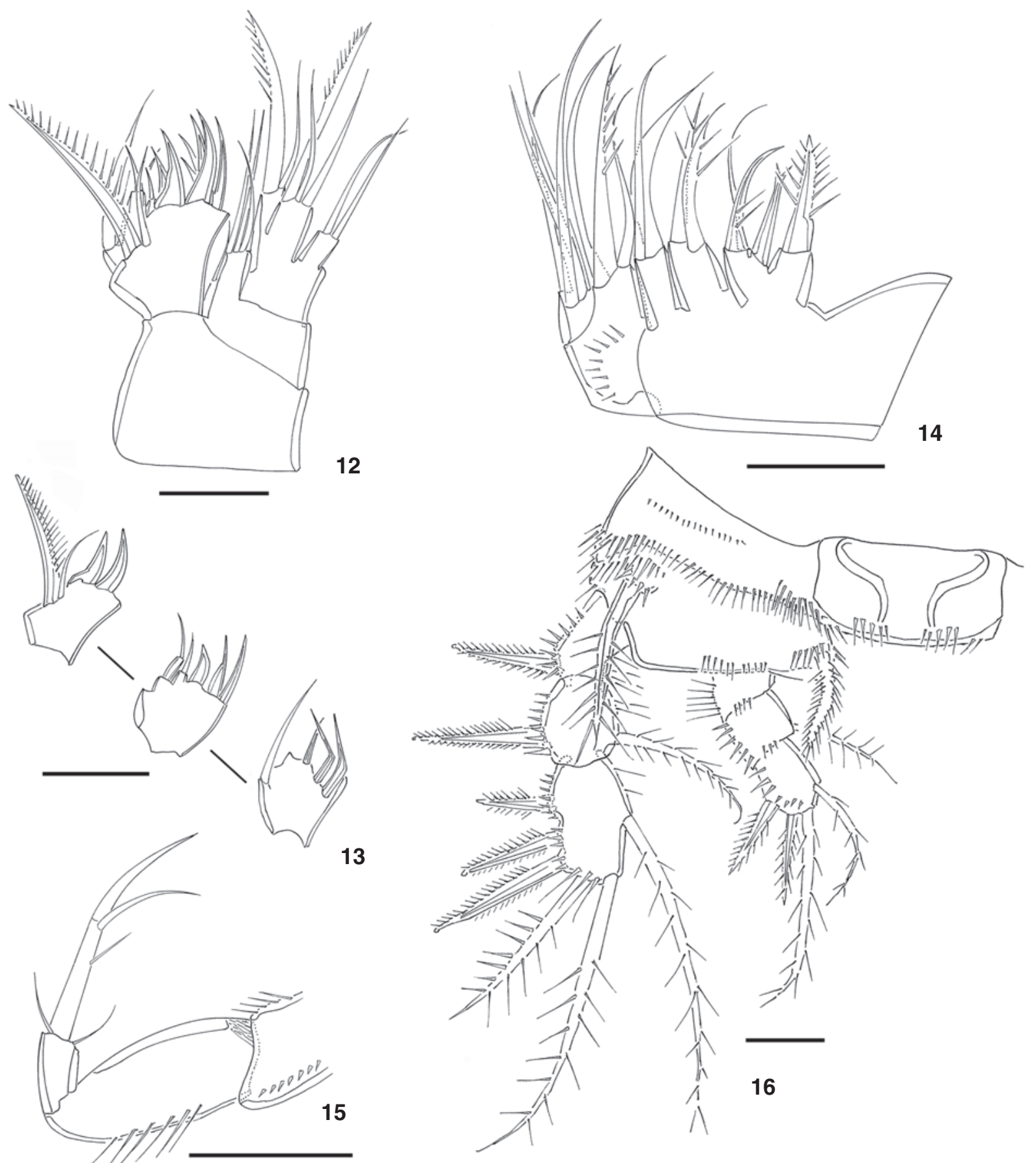

Figuras 12-16. Microarthridion corbisierae sp. nov., fêmea: (12) maxílula; (13) artrito da maxílula em diferentes planos; (14) maxila; (15) maxilípede; (16) perna 1, anterior. Escala $=10 \mu \mathrm{m}$.

lisas. Coxa com quatro cerdas lisas. Endito da base com uma cerda lisa e outra unipinada; exópodo e endópodo fusionados à base. Exópodo com duas cerdas lisas. Endópodo com duas cerdas lisas e uma unipinada.
Maxila (Fig. 14). Sincoxa com três enditos; endito proximal bilobado, com dois espinhos robustos e pinados, um espinho de ápice bífido e uma cerda lisa; endito médio com duas cerdas pinadas; endito distal com duas cerdas lisas. Alobase 


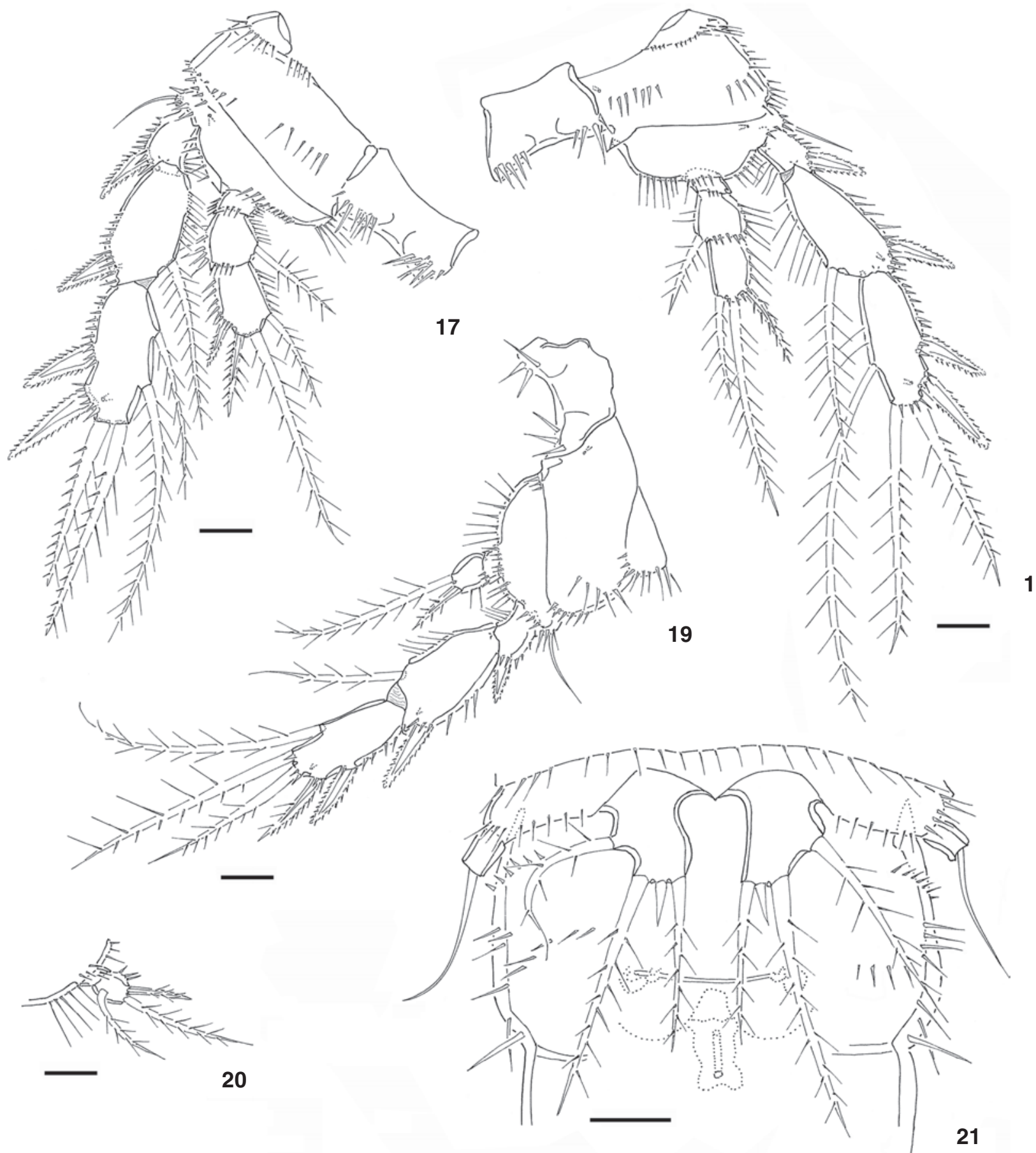

Figuras 17-21. Microarthridion corbisierae sp. nov., fêmea: (17) perna 2, anterior; (18) perna 3, anterior; (19) perna 4 esquerda, anterior; (20) endópodo da perna 4 direita desarticulado, mesmo indivíduo, anterior; (21) vista ventral do urossomo-1 e parte anterior do segmento genital duplo com par de pernas 5 e área genital, respectivamente. Escala $=10 \mu \mathrm{m}$.

com fileira curva de espínulos, duas cerdas lisas e uma cerda robusta unipinada. Endópodo com quatro cerdas lisas.

Maxilípede (Fig. 15) sub-quelado. Sincoxa e base com fi- leiras de espínulos. Endópodo uniarticulado, com duas cerdas reduzidas e lisas e, com garra apical pinada.

Perna 1 (Fig. 16). Placa intercoxal com uma fileira de 

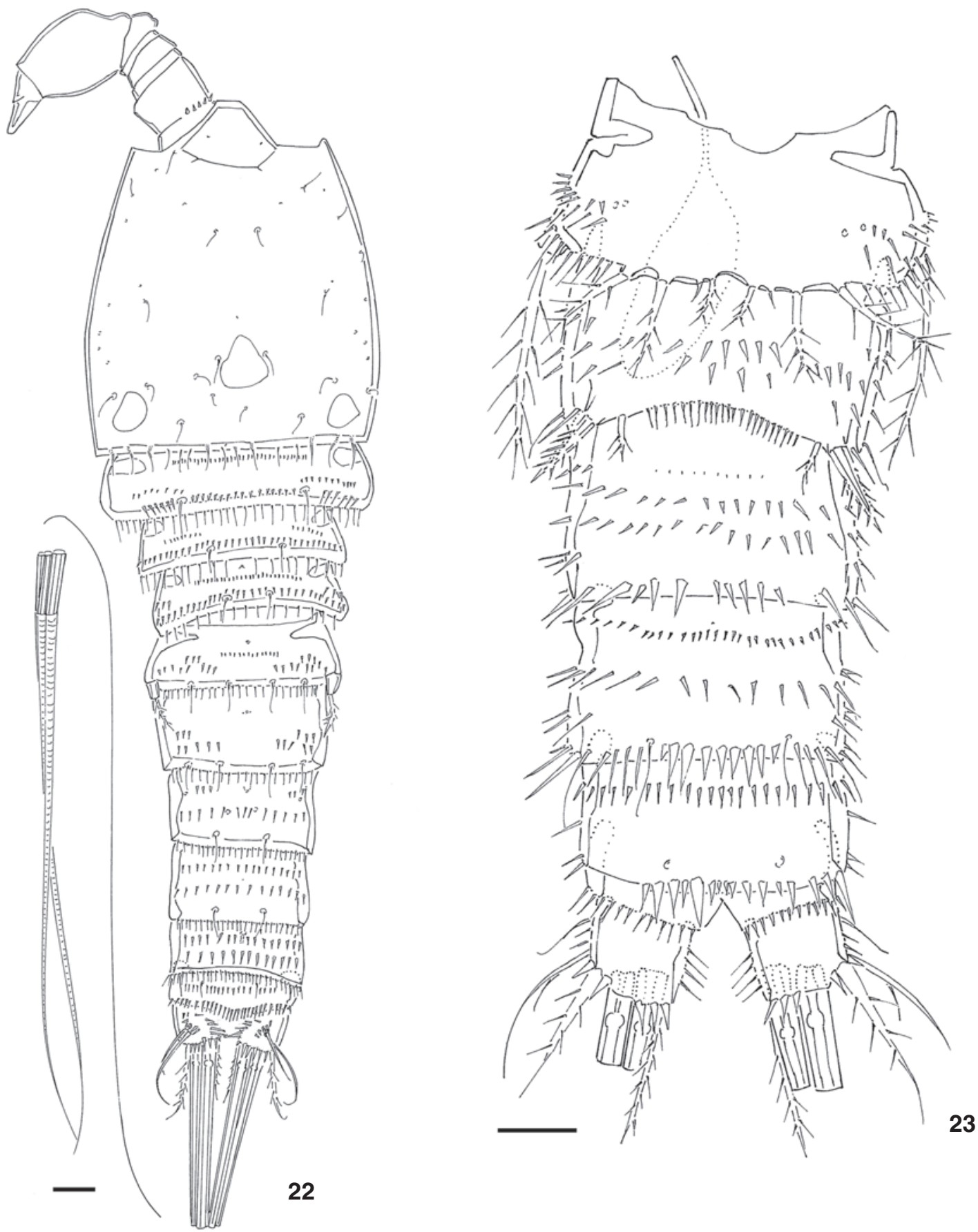

Figuras 22-23. Microarthridion corbisierae sp. nov., macho: (22) hábito, dorsal; (23) urossomo, ventral (espermatóforo representado em linha pontilhada na porção anterior do urossomo). Escala $=10 \mu \mathrm{m}$.

espínulos de cada lado da margem distal. Coxa com fileira de espínulos na porção média da superfície anterior e outra ao longo do bordo distal. Base com fileiras de espinhos; cerda externa bipinada; espinho interno robusto e com plumosidades.
Exópodo com três artículos; fileiras de espínulos ao longo das margens interna e externa; espinhos robustos e bipinados; exópodo-1 sem cerda interna; exópodo- 2 com cerda plumosa; exópodo-3 com 3 espinhos externos serrilhados, duas cerdas 


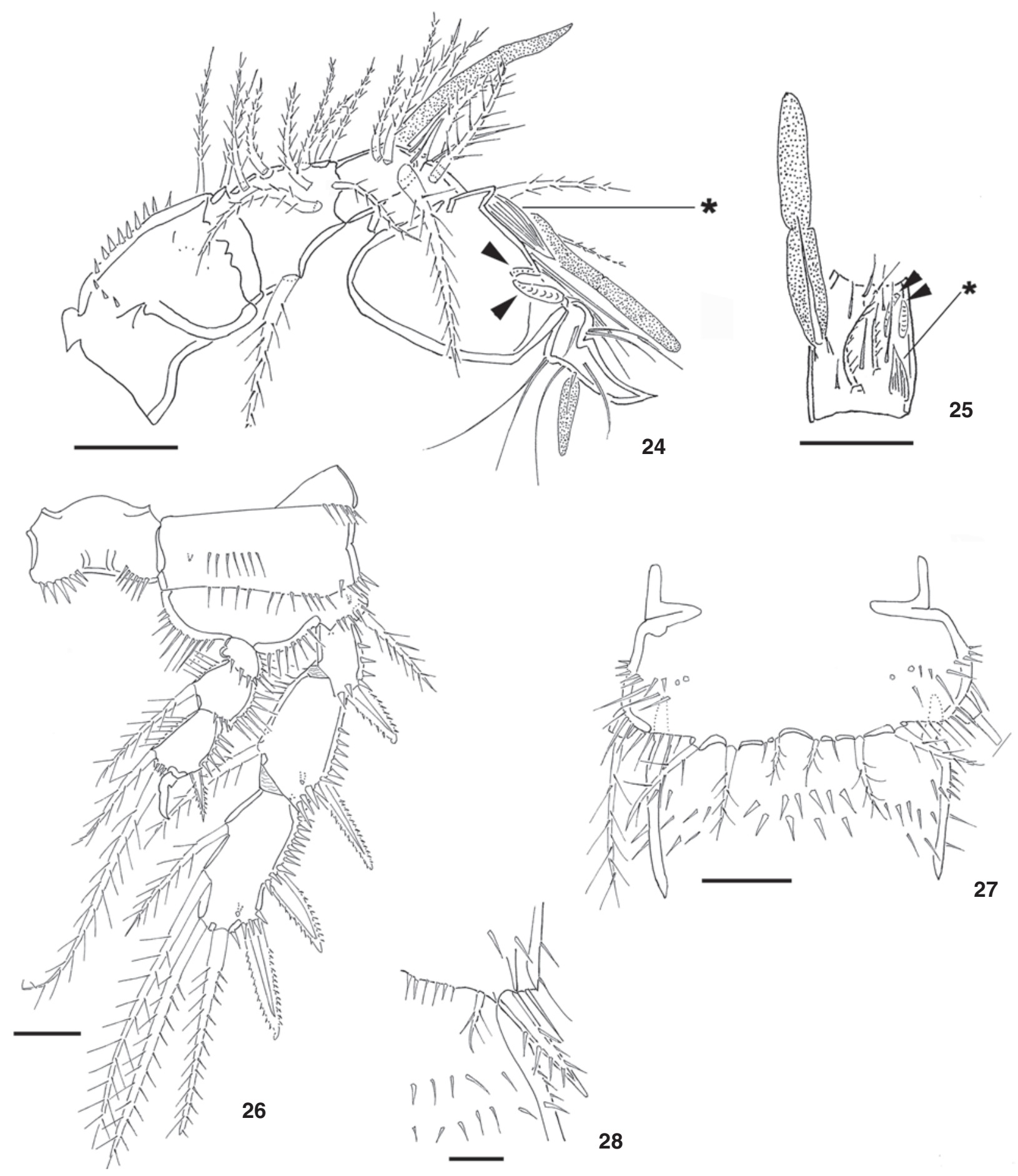

Figuras 24-28. Microarthridion corbisierae sp. nov., macho: (24) antênula, dorsal (setas indicando elementos com ranhuras transversais e asterisco indicando cerda laminar); (25) artículo 6 da antênula, anterior (setas indicando elementos com ranhuras transversais e asterisco indicando cerda laminar); (26) perna 2, anterior; (27) urossomo-1 com par de pernas 5 e parte anterior do segmento genital, ventral; (28) canto posterior esquerdo do segmento genital portando a perna 6, ventral. Escala $=10 \mu \mathrm{m}$. 
bipinadas e uma cerda plumosa. Endópodo com três artículos; fileiras de espínulos ao longo das margens externas e distais; endópodo-1 curto, sem cerda interna; endópodo- 2 com cerda plumosa; endópodo-3 com duas cerdas plumosas e dois espinhos bipinados.

Pernas 2-4 (Figs 17-20). Placa intercoxal com grupos de espínulos próximos aos cantos distais. Pré-coxa com fileira de espínulos delgados. Coxa com fileira transversal de espínulos na superfície anterior. Base com fileiras transversais de espínulos delgados ao longo do bordo distal; cerda externa lisa. Exópodo triarticulado; cada artículo com espínulos ao longo da margem externa e cérdulas na margem interna; espinhos externos robustos e serrilhados; exópodo-1 sem cerda interna; exópodo-2 com cerda interna plumosa; exópodo-3 com dois espinhos externos, duas cerdas apicais bipinadas e uma (pernas 3 e 4) ou duas (perna 2) cerdas plumosas. Endópodo triarticulado nas pernas 2 e 3 , e biarticulado na perna 4; fileiras de espinhos nas margens distal e externa de cada artículo; endópodo- 1 muito reduzido, sem cerda interna; endópodo- 2 das pernas 2 e 3 com uma cerda plumosa cada; endópodo-3 da perna 2 com duas cerdas plumosas e dois espinhos bipinados; endópodo-3 da perna 3 com duas cerdas plumosas e um espinho bipinado; último artículo do endópodo da perna 4 com um espinho e uma ou duas cerdas plumosas no mesmo indivíduo (Figs 19 e 20). Fórmula de cerdas e espinhos como se segue: Perna1 - Exópodo: 0.1.123, Endópodo: 0.1.121; Perna 2 - Exópodo: 0.1.222, Endópodo: 0.1.121; Perna 3 Exópodo: 0.1.122, Endópodo: 0.1.021; Perna 4 - Exópodo: 0.1.122, Endópodo: 0.0-120.

Perna 5 (Fig. 21). Cerda basal externa lisa e inserida sobre protuberância disposta lateralmente. Artículo retangular com três cerdas longas e bipinadas e um espinho liso.

Fêmea com um saco de ovos.

Descrição. Macho (Figs 22-28). Comprimento total do corpo medido da extremidade do rostro até a margem posterior do ramo caudal 210-0230 $\mu \mathrm{m}(\mathrm{n}=4$, média $=220)$. Prossomo:urossomo 1,3-1,7:1 ( $\mathrm{n}=4$, média $=1,6: 1$ ). Segmentos com espínulos mais robustos e dispostos em um menor número de fileiras que aqueles da fêmea (Figs 22 e 23).

Antênula (Figs 24 e 25) curta, com sete artículos, quirócera. Geniculação entre artículos 6 e 7 presente; artículo1 parcialmente dividido, com fileiras de espínulos curtos e robustos; artículo- 6 dilatado, com dois elementos com ranhuras transversais incorporados ao artículo, uma cerda laminar (Figs 24 e 25). Fórmula de cerdas e estetos: 1, 1, 9, 7 + esteto, 3, $10+$ 2 elementos com ranhuras + esteto, $8+$ esteto.

Perna 2 (Fig. 26). Base com fileiras de espínulos delgados; cerda externa robusta e bipinada. Exópodo similar ao da fêmea. Endópodo com três artículos; endópodo-2 sem apófise; endópodo-3 com um espinho em forma de gancho.

Par de pernas 5 (Figs 23, 27) fundidas entre si na região mediana e incorporadas ao segmento; cada perna 5 com uma cerda bipinada, duas cerdas plumosas e uma cerda lisa muito reduzida; cerda basal externa plumosa, disposta lateralmente.
Par de pernas 6 (Figs 23, 28) simétrico; cada uma das pernas com uma cerda interna plumosa, um espinho mediano bipinado e robusto, e uma cerda externa lisa.

Etimologia. A espécie é dedicada à Dra Thaïs Corbisier (Instituto Oceanográfico da Universidade de São Paulo), por sua contribuição ao estudo da meiofauna no Brasil.

\section{DISCUSSÃO}

Embora compartilhe com M. laurenticum (Nicholls, 1940) a presença de apenas cinco artículos na antênula, a nova espécie pode ser facilmente diferenciada de seus congêneres pela redução no número de artículos do endópodo da perna 4 de três para dois. Uma outra característica que a distingue das demais espécies é a redução no número de cerdas da margem interna do endópodo-3 das pernas 1-4 e do exópodo-3 da perna 3 (Tab. I).

Ampliando a comparação para todos os gêneros da família Tachidiidae, a nova espécie apresenta, junto com Cithadius, endópodo da perna 4 biarticulado (Bowman 1972). Entretanto, é provável que a existência de dois artículos nos dois ramos da perna 4 de Cithadius tenha evoluído de forma independente, como adaptação à vida associada aos isópodes.

Pelas características observadas nas pernas natatórias, foi suposto inicialmente que os espécimes aqui estudados deveriam ser separados em um novo gênero. Nossa decisão foi revista, levando em conta a pequena quantidade de espécimes à disposição e a informação prestada pelo Dr R. Huys, The Natural History Museum, London, que teve a oportunidade de estudar vários indivíduos da família Tachidiidae, alguns ainda não descritos, de que a variabilidade nos caracteres de Microarthridion é significativa e poderia permitir a inclusão desse novo táxon encontrado no Brasil.

Copépodes Tachidiidae têm sido, desde o relato de BoEck (1865), coletados e descritos de várias localidades do Hemisfério Norte. Esta primeira ocorrência da família no Brasil e registros não publicados da Nova Zelândia mencionados por Huys et al. (2005) indicam que Tachidiidae não deve possuir uma distribuição geográfica preferencial e abrem futuras áreas de pesquisa no Brasil, onde a visitação a novas localidades para coleta e estudo taxonômico dos copépodes harpacticóides da meiofauna marinha encontra-se apenas no início.

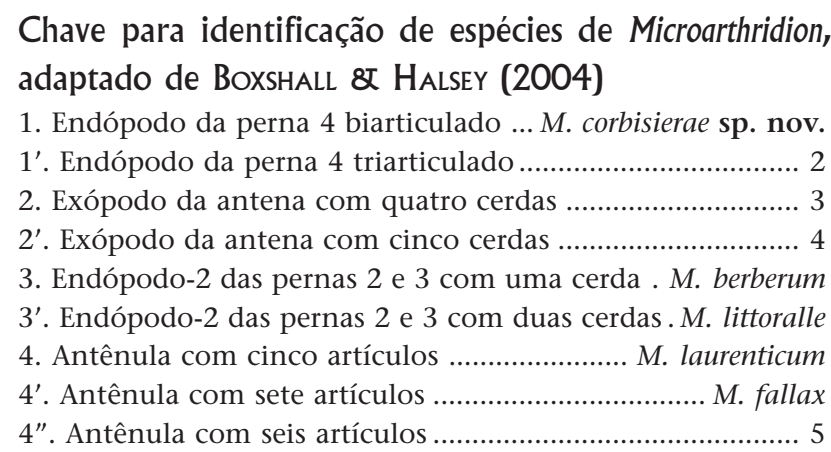


Tabela I. Fórmula de cerdas e espinhos de representantes de Microarthridion segundo notação proposta por HuYs et al. (1996). Compilado de Monard (1935), Nicholls (1940), Lang (1944), Bodin (1970), Shen \& Tal (1973) e Huys et al. (1996).

\begin{tabular}{|c|c|c|c|c|c|c|c|c|}
\hline \multirow{2}{*}{ Espécie } & \multicolumn{2}{|c|}{ Perna 1} & \multicolumn{2}{|c|}{ Perna 2} & \multicolumn{2}{|c|}{ Perna 3} & \multicolumn{2}{|c|}{ Perna 4} \\
\hline & Exópodo & Endópodo & Exópodo & Endópodo & Exópodo & Endópodo & Exópodo & Endópodo \\
\hline M. berberum (Monard, 1936) & 0.1 .123 & 0.1 .221 & 0.1 .222 & 0.1 .221 & 0.1 .322 & 0.1 .321 & 0.1 .122 & 0.1 .221 \\
\hline M. fallax Perkins, 1956 & 0.1 .123 & 0.1 .221 & 0.1 .222 & 0.1 .221 & 0.1 .222 & 0.1 .321 & 0.1 .122 & 0.1 .221 \\
\hline M. laurenticum (Nicholls, 1940) & 0.1 .123 & 0.1 .221 & 0.1 .222 & 0.2 .221 & 0.1 .222 & 0.2 .321 & 0.1 .122 & 0.1 .221 \\
\hline M. litospinatus Shen \& Tai, 1973 & 0.1 .123 & 0.1 .221 & 0.1 .222 & 0.2 .221 & 0.1 .222 & 0.2 .321 & 0.1 .122 & 0.1 .221 \\
\hline M. littorale (Poppe, 1881) & 0.1 .123 & 0.1 .221 & 0.1 .222 & 0.2 .221 & 0.1 .222 & 0.2 .321 & 0.1 .122 & 0.1 .221 \\
\hline M. perkinsi Bodin, 1970 & 0.1 .123 & 0.1 .221 & 0.1 .222 & 0.2 .221 & 0.1 .222 & 0.2 .321 & 0.1 .122 & 0.1 .221 \\
\hline M. reductum (Monard, 1935) & 0.1 .123 & 0.1 .221 & 0.1 .222 & 0.2 .221 & 0.1 .222 & 0.2 .321 & 0.1 .122 & 0.1 .221 \\
\hline M. corbisierae sp. nov. & 0.1 .123 & 0.1 .121 & 0.1 .222 & 0.1 .121 & 0.1 .122 & 0.1 .021 & 0.1 .122 & $0.0-120$ \\
\hline
\end{tabular}

5. Endópodo-2 da perna 1 com uma cerda interna reduzida .. ... . reductum

5'. Endópodo-2 da perna 1 com uma cerda interna bem desenvolvida 6

6. Exópodo da perna 5 com três cerdas longas e plumosas; e uma cerda curta e lisa .................................. M. litospinatus

6'. Exópodo da perna 5 com quatro cerdas longas e plumosas M. perkinsi

\section{AGRADECIMENTOS}

À Thaïs N. Corbisier, do Departamento de Oceanografia Biológica, Instituto Oceanográfico (IOUSP), por ter colocado o material coletado durante a realização do projeto multidisciplinar "Utilização Racional do Ecossistema Costeiro da Região Tropical Brasileira: Estado de São Paulo" a nossa disposição para estudo taxonômico, e à equipe que ofereceu e auxiliou na disciplina "Ecologia de micro e meiobentos marinhos" do Programa de Pós-graduação em Oceanografia Biológica (IOUSP). Também o apoio financeiro da Coordenadoria de Pós-graduação, Pesquisa e Extensão do Centro Universitário Adventista de São Paulo (UNASP), do CNPq e do Programa BIOTA/FAPESP - Bentos Marinho (Proc. 1998/07090-3). O trabalho é parte da tese de doutorado de Terue C. Kihara e foi realizado com o apoio da CAPES.

\section{REFERÊNCIAS BIBLIOGRÁFICAS}

Bodin, P. 1970. Copépodes Harpacticoïdes marins des environs de La Rochelle. 1. Espèces de la vase intertidale de Chatelaillon. Tethys 2 (2): 385-436.

Bовск, A. 1865. Oversigt over de ved Norges Kyster jagttagne Copepoder henhörende til Calanidernes, Cyclopidernes og Harpactidernes Familier. Forhandlinger i Videnskabsselskabet i Kristiania 1864: 226-282.

Bowman, T.E. 1972. Cithadius cyathurae, a new genus and species of Tachidiidae (Copepoda: Harpacticoida) associated with the estuarine isopod, Cyathura polita. Proceedings of the Biological Society of Washington 85 (18): 249-254.
Boxshall, G.A. \& S.H. Halsey. 2004. An introduction to copepod diversity. London, The Ray Society, 966p.

Corbisier, T.N. 1993. Meiofauna da plataforma continental interna do litoral norte de São Paulo - verão/89. Publicação Especial do Instituto Oceanográfico 10: 123-135.

Dussart, B. 1967. Les Copépodes des eaux continentales d'Europe occidentale. Tome I: Calanoïdes et Harpacticoïdes. Paris, N. Boubée Ed., 500p.

FurTADO, V.V. \& M.M. MAHIQUES. 1990. Distribuição de sedimentos em regiões costeiras e plataforma continental norte do Estado de São Paulo. Simpósio de Ecossistemas da Costa Sul e Sudeste Brasileira: estrutura, função e manejo 2 (1): 20-29.

Huys, R.; J.M. Gee; C.G. Moore \& R. Hamond. 1996. Marine and brackish water harpacticoid copepods. Part 1. In: D.M. Kermack; R.S.K. Barnes \& J.H. Crothers (Eds). Synopses of the British Fauna, New series: 1-352.

Huys, R.; S. OHTSuKa; S. Conroy-Dalton \& Y. Kiruchi. 2005. Description of two new species of Neotachidius Shen \& Tai, 1963 (Copepoda, Harpacticoida, Tachidiidae) from Korean brackish waters and proposal of a new genus for Tachidius (Tachidius) vicinospinalis Shen \& Tai, 1964. Zoological Journal of the Linnean Society 143 (1): 133-159.

LANG, K. 1944. Monographie der Harpacticiden (Vorlaufige Mitteilung). Uppsala, Almqvist \& Wiksells Boktryckeri Ab, 39p.

Lorenzen, S. 1969. Harpacticoiden aus dem lenitischen Watt und den Salzwiesen der Nordseeküste. Kieler Meeresforschungen 25 (1): 215-223.

Monard, A. 1935. Étude sur la faune des Harpacticoides marins de Roscoff. Travaux de la Station Biologique de Roscoff 13: 5-88.

Nicholls, A.G. 1940. Marine harpacticoids and cyclopoids from the shores of the St. Lawrence. Fauna et Flora Laurentianae 2: 241-316.

Pires-Vanin, A.M.S. \& Y. Matsuura. 1993. Estrutura e função do escossistema de plataforma continental da região de Ubatuba, Estado de São Paulo, Estado de São Paulo: uma introdução. Publicação Especial do Instituto Oceanográfico 10: 1-8. 
SeIfried, S. 2003. Phylogeny of Harpacticoida (Copepoda): revision of Maxillipedasphalea and Exanechentera. Göttingen, Cuvillier Verlag, 259p.

Shen, C.J. \& A.Y. TAI. 1963. On five new species, a new subgenus and a new genus of freshwater Copepoda (Harpacticoida) from the delta of the Pearl River, South China. Acta Zoologica Sinica 15 (3): 417-432.

Shen, C.J. \& A.Y. Tai. 1973. Preliminary analysis of the charac-

Recebido em 18.VI.2007; aceito em 12.XI.2007. teristics of the harpacticoid copepod fauna of China and description of some new species. Acta Zoologica Sinica 19 (4): 365-384.

Veldre, I. \& A. Maemets. 1956. Eesti NSV vabaltevad aerjalalised (Eucopepoda) II. Soudikulised (Cyclopoida), Rullikulised (Harpacticoida). Die freilebenden Ruderfüssler der estnischen SSR, II. (Cyclopoida, Harpacticoida). Abiks Loodusevaatlejale. Akadeemia Juures 29: 1-128. 